\title{
Identificación de áreas de aplicación de arquitecturas de software basadas en modelos, técnicas y herramientas de social media
}

\author{
Paola E. Velazquez-Solis ${ }^{1}$, Brenda L. Flores-Rios ${ }^{1}$, Jorge E. Ibarra-Esquer ${ }^{2}$, \\ María Angélica Astorga-Vargas², Raúl A. Aguilar Vera ${ }^{3}$, Elizabeth Ramírez-Barreto \\ Gabriel López-Morteo ${ }^{1}$
}

\{paola.velazquez; brenda.flores; jorge.ibarra; angelicaastorga; eramirez; galopez\} @uabc. edu.mx; avera@correo.uady.mx

${ }^{1}$ Instituto de Ingeniería, 2 Facultad de Ingeniería, Universidad Autónoma de Baja California, Mexicali, Baja California, México.

${ }^{3}$ Facultad de Matemáticas, Universidad Autónoma de Yucatán, Mérida, México.

DOI: 10.17013/risti.42.12-29

\begin{abstract}
Resumen: Social media se refiere al conjunto de técnicas y herramientas que están tomando relevancia para la recolección de información sobre el comportamiento de los usuarios en las redes sociales. El objetivo de esta investigación fue identificar los enfoques, modelos de análisis, técnicas y herramientas de social media aplicados en arquitecturas de software, con el fin de detectar las áreas de aplicación por medio de estudios empíricos. Para ello, se realizó un mapeo sistemático de la literatura con el cual se seleccionaron treinta artículos publicados entre 2015 y 2020. Los resultados validaron que en los últimos tres años se han incrementado en un 57\% las publicaciones, principalmente en el área de investigación centrándose en diseñar arquitecturas de software que incorporen modelos de análisis de social media en los componentes y crear nuevas oportunidades en las redes sociales.
\end{abstract}

Palabras-clave: social media; arquitecturas de software; redes sociales; mapeo sistemático de la literatura

\section{Identification of application areas of software architectures based on social media models, techniques and tools}

Abstract: Social media refers to the set of techniques and tools that are gaining relevance for the collection of information about the behavior of users on social networks. The objective of this research was to identify the approaches, analysis models, techniques, and tools of social network applications in software architectures, to detect the application areas through empirical studies. For this, a systematic mapping of the literature was carried out, with which thirty articles published between 2015 and 2020 were selected. The results validated that in the last three years publications have increased by $57 \%$, mainly in the research area focusing on designing software architectures that incorporate social network analysis models in the components and create new opportunities in social networks. 
Keywords: social media; software architectures; social networks; systematic literature mapping

\section{Introducción}

En una red social las personas o grupos de personas interactúan entre sí en un sitio de Internet. Las redes sociales se caracterizan por tener una base de usuarios amplia y heterogénea, con participación tanto intermitente como constante, convirtiéndose en un medio imprescindible de comunicación e información, para la creación de proyectos, integración de comunidades y nuevas formas de entretenimiento y acción social colectiva (Ureña et al., 2019). La incorporación de técnicas y criterios de análisis propios de social media en las redes sociales, tiene como propósito el de mantener una comunicación ubicua al crear y compartir contenidos (Martín Blanco, 2015). De esta forma, social media se refiere a un conjunto de técnicas, herramientas, aplicaciones y medios de comunicación donde la información es creada y visualizada por los usuarios, que son libres de compartir y transferir textos, fotografías, audio, vídeo, entre otro tipo de contenido (Injadat et al., 2016). Las aplicaciones más comunes de social media incluyen: monitorear las respuestas públicas a anuncios o discursos, la difusión de eventos, analizar comentarios e iniciativas políticas, identificar ideas u opiniones sobre el comportamiento de una comunidad, encuestar grupos de redes sociales o detectar la aparición temprana de eventos emergentes - conocido también como la viralización de contenidos (Batrinca \& Treleaven, 2015). Las áreas estratégicas que pueden monitorearse en social media son las necesidades, los sentimientos y la comunicación entre los usuarios. Este seguimiento se puede realizar directamente en redes sociales como Facebook (Kurnia \& Suharjito, 2018), Twitter o en otros ámbitos los cuales incluyen áreas de negocios, educación, gobierno o salud. Sin embargo, el desarrollo de herramientas o aplicaciones que se apoyen de una metodología enfocada a social media es una necesidad inminente (Al-Maatouk et al., 2020; Diamantini et al., 2019; Mushtaq et al., 2018; Verhoef \& Lemon, 2013).

Las redes sociales, social media y los espacios virtuales son mecanismos de interacción o canales de comunicación, con un alcance y potencial de mayor impacto que cualquiera de los otros medios tradicionales, y con capacidad de interacción y dinamismo superior (Herrera, 2012). Los servicios de redes sociales se han vuelto cruciales para algunas organizaciones en el servicio al cliente o en sus departamentos de mercadotecnia donde se proponen soluciones para la indexación de influencers ${ }^{1}$ o nichos de oportunidad para incursionar dentro del Internet (Arora et al., 2019). También, presentando estrategias de publicación eficaz dentro de las plataformas de redes sociales, la identificación del tipo de mensajes o los tipos de transporte de mensajes apropiados (Bitiktas \& Tuna, 2020). Por otro lado, en el estudio de Rousidis, Koukaras \& Tjortjis (2020) en sus hallazgos indicaron que los docentes o académicos de cualquier disciplina o cultura, podrían impartir cursos a través de plataformas como redes sociales gracias a las diferentes funcionalidades que brindan las herramientas de social media, como Portal Home, Historias de voz y el almacenamiento en la nube de Facebook, los cuales incentivaban a

${ }^{1}$ Persona(s) que destaca(n) en una red social u otro canal de comunicación y expresa opiniones sobre un tema concreto que ejercen una gran influencia sobre muchas personas que la conocen. 
los estudiantes a realizar una participación más activa. Esto de alguna forma propicia la modificación de las prácticas tradicionales o los contenidos temáticos de los programas de enseñanza-aprendizaje en las instituciones educativas. En ambas áreas de aplicación la comunicación siempre se estable con los usuarios.

En el ámbito de la Ingeniería de Software, existen propuestas sobre arquitecturas (Raspopović et al., 2016) basadas en social media, incluso, ciertos autores (Hayat et al., 2019; Peláez et al., 2019) han propuesto metodologías para el diseño de arquitecturas que apoyen el descubrimiento de información social, considerando como requisitos: la velocidad de la transmisión de datos, la flexibilidad y la escalabilidad (Diamantini et al., 2019). Diamantini et al. (2019) proponen una arquitectura para social media Data Analytics de manera que sea posible construir diferentes sistemas para analizar diversas redes sociales con base en la arquitectura. Sin embargo, dicho trabajo es una implementación, sin entrar en detalles sobre la analítica de datos, utilizando algoritmos para clasificar los sentimientos de los usuarios. El proceso descrito por Mushtaq et al. (2018) se realiza desde la perspectiva de los datos en bruto en las redes sociales, y es el interés particular, el que permite llegar a una clasificación (p.e. conocer relaciones y asociaciones entre publicaciones). Algunos trabajos tienen el dominio de desarrollo dentro del Aprendizaje profundo o Minería de datos, lo cual a pesar de ser una aproximación más real a los datos obtenidos desde las redes sociales, debido a que difumina una mejor visión de dominios y aplicaciones futuras (Hayat et al., 2019), no permite tener certeza de la interoperabilidad, seguridad y portabilidad de las arquitecturas propuestas.

De acuerdo con lo antes expuesto, se considera importante analizar las técnicas de social media que sea parte de un marco de referencia para la construcción de software, con arquitecturas de software adecuadas para social media. El objetivo del presente estudio se centra en identificar modelos de análisis, técnicas y herramientas de social media aplicadas en arquitecturas de software propuestos en diversas áreas de aplicación, a partir de la implementación de un mapeo sistemático de literatura.

Este documento se estructura de la siguiente manera: en la sección 2 se introduce un marco referencial sobre los términos de redes sociales, social media y arquitectura de software. La sección 3 especifica el protocolo metodológico utilizado; la sección 4 presenta las áreas de aplicación identificadas en los resultados obtenidos a partir del mapeo sistemático de literatura realizado. Finalmente, la sección 5 presenta las conclusiones.

\section{Marco de Referencia}

Las redes sociales son servicios basados en Web que permiten a las personas (Peruta \& Shields, 2017): 1) construir un perfil público o semipúblico dentro de un sistema; 2) crear una lista de otros usuarios con los que se comparte una conexión y contenido; 3) conocer y recorrer un conjunto de datos referente a las conexiones y contenidos realizados por otros dentro del sistema. De esta forma, una red social es una herramienta que ha transformado a las personas en receptores y productores de contenido (Gonzalez \& Munoz, 2016) propiciando nuevos métodos de comunicación entre ellos para tomar decisiones, socializar, colaborar, aprender, realizar compras e interactuar entre ellos (Sabate et al., 2014). 
Los datos generados por las redes sociales son una clara evidencia de la dinámica del comportamiento humano, los cuales brindan nuevas oportunidades para estudiar y analizar su socialización. Por ejemplo, entre grupos escolares, académicos, deportivos o la sociedad misma. Tanto científicos, profesionales como responsables de las organizaciones han identificado nuevas formas de recopilar, combinar y analizar automáticamente la información contenida en las grandes bases de datos desde una perspectiva organizacional (Batrinca \& Treleaven, 2015). Últimamente, se han desarrollado aplicaciones basadas en modelos de Minería de datos con el propósito de realizar el monitoreo del desempeño de las publicaciones en las redes sociales, reduciendo el tiempo de espera para obtener métricas o estadísticas de páginas como Facebook o Twitter (Kurnia \& Suharjito, 2018 y Purohit et al., 2020). Los modelos utilizados se basan en la extracción del conocimiento de los conjuntos de datos, para posteriormente analizarlos con el objetivo de comprender no solo el comportamiento social virtual, sino también los sentimientos y emociones de quienes interactúan (He et al., 2017; Salloum et al., 2017 y Tocoglu et al., 2019).

Según Lara (2008) existen cinco requisitos que deben de cumplir los medios digitales para consolidar sus redes sociales desde un enfoque de social media: conectividad con el usuario, servicios útiles para los usuarios, participación abierta y de calidad, orientación y dinamización y una Gestión del conocimiento. De esta forma, es más común encontrar usuarios con los cuales se compartan gustos, aficiones, fotografías, contenido tanto de video como de audio (Martin Blanco, 2015), por lo que la participación de usuarios en las redes sociales con social media se espera que exista una cultura colaborativa con grados o niveles de participación, definiendo cursos de acción y diferenciando sus roles. El uso de técnicas de social media, en los tweets y retweets de Twitter y los compartir (share) de Facebook, se convirtieron en una área de investigación computacional y de negocios debido al rápido cambio y evolución de las actividades comerciales y la disponibilidad de Interfaces de Programación de Aplicaciones (API por sus siglas de Application Programming Inteface) basadas en la Web proporcionadas por Twitter, Facebook y los servicios de noticias (Batrinca \& Treleaven, 2015).

Por consiguiente, se ha generado un crecimiento de servicios de datos y la necesidad de incorporar técnicas y herramientas de social media en el desarrollo de arquitecturas de software. En Ingeniería de software, el concepto de arquitectura se refiere a la estructura organizativa de un sistema o componente. También al proceso de definir una colección de componentes de hardware y software, las interfaces para establecer el marco para el desarrollo de un sistema informático y las relaciones de trabajo entre los componentes de un sistema (Maier et al., 2001). Algunos diseños de arquitectura de software son: microservicios, por capas y componentes. Los enfoques de los autores varían, en relación al área de aplicación de la arquitectura y los elementos necesarios en los requerimientos. Hayat et al. (2019) exponen la arquitectura de forma genérica, indicando los diversos componentes, tales como métodos, módulos y algoritmos.

\section{Metodología}

De acuerdo con el objetivo del estudio, se determinó utilizar un mapeo sistemático de la literatura siguiendo los lineamientos de (Petersen et al., 2015). El proceso consta de 
cinco pasos para obtener el estado del arte en el tema analizado (Brenes Carranza et al., 2020; Ramírez-Alpízar et al., 2020).

\subsection{Preguntas de Investigación}

Para conducir el estudio se utilizó el modelo PICO (Población, Intervención, Comparación, Salidas) (Wohlin et al., 2012) y se plantearon las siguientes Preguntas de Investigación (PI):

- PI1. ¿Cuáles enfoques y modelos se han considerado en el desarrollo de arquitecturas de software para social media?

- $\quad$ PI2. ¿Qué técnicas y/o herramientas de social media se han utilizado en el diseño de arquitecturas de software?

Con el propósito de realizar una búsqueda exploratoria basada en el objetivo y las PI, se construyó una cadena de búsqueda. La búsqueda exploratoria permitió identificar los estudios base que fueron guía para el proceso de búsqueda (Bitiktas \& Tuna, 2020; Chugh \& Ruhi, 2018; Peruta \& Shields, 2017; Purohit, Castillo \& Pandey, 2020).

\subsection{Estrategia de búsqueda y proceso de selección de estudios}

La cadena de búsqueda se construyó en función del contenido de términos clave del título de este estudio y los criterios de selección de los autores.

("Software Architecture" AND "social media" OR "social media analytics" OR "social media mining" OR "Sentiment analysis" OR “Opinion mining”)

La Figura 1 presenta el proceso de búsqueda utilizando como fuentes las bases de datos IEEE Electronic Library (IEL), Elsevier, Springer Link, Scopus y agrupado como otras fuentes Google Scholar, AMC y Wiley. Se buscaron los términos de la cadena contra el título, el resumen, las palabras clave y las conclusiones de los artículos. El protocolo base del mapeo fue desarrollado durante el mes de septiembre de 2020. La búsqueda

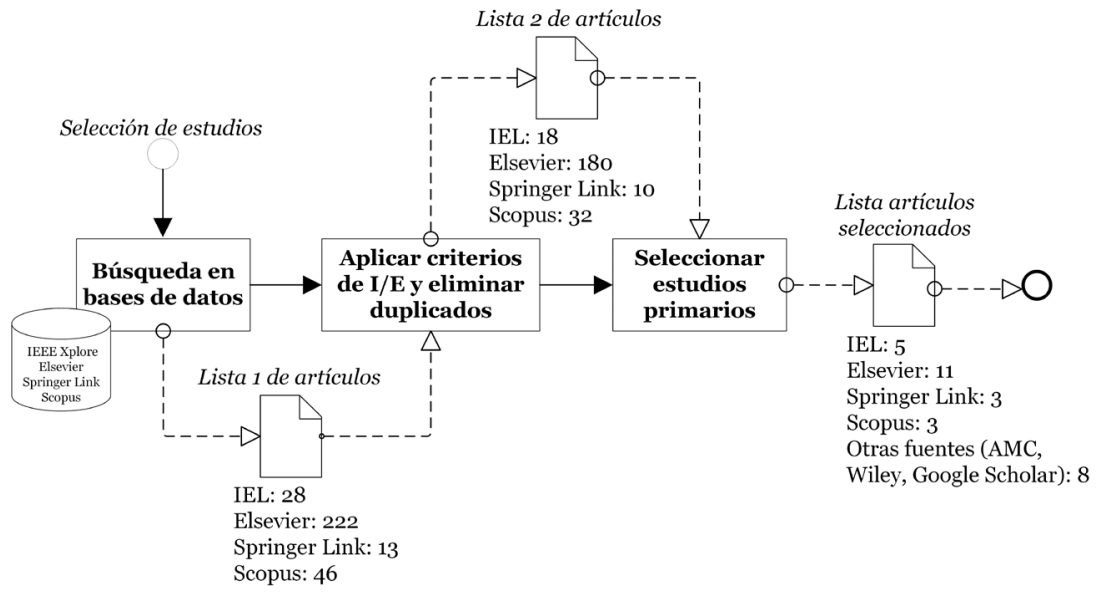

Figura 1 - Proceso de selección de los artículos. Elaboración propia. 
automatizada se realizó en octubre y los estudios se analizaron entre noviembre y diciembre de ese mismo año. El número de artículos que se recuperaron de cada base de datos fueron: 28 en IEL, 46 en Scopus, 13 en Springer Link, 222 en Elsevier y 11,100 en Google Scholar, considerando AMC y Wiley.

Los criterios de I/E se aplicaron en el título y resumen de los artículos. Se incluyeron los que cumplían con ((I1 OR I2) AND I3 AND I4 AND I5) y Se excluyeron aquellos artículos que cumplían con la fórmula (E1 OR E2 OR E3), como se presenta en la Tabla 1. A partir de esta estrategia de búsqueda y el proceso de selección expuestos, se eliminaron los artículos duplicados quedando seleccionados 30 estudios primarios.

\begin{tabular}{ll}
\hline Criterios de inclusión & Criterios de exclusión \\
\hline I1: Bases de datos científicas seleccionadas. & $\begin{array}{l}\text { E1: Artículos de memorias de congresos, tesis, } \\
\text { revistas no arbitradas, workshops. }\end{array}$ \\
\hline I2: Publicaciones científicas indexadas. & $\begin{array}{l}\text { E2: Estudios que no estén disponibles en texto } \\
\text { completo. }\end{array}$ \\
\hline I3: Publicaciones entre 2015 y 2020. & E3: Estudios terciarios y secundarios. \\
\hline I4: Estudios en idioma inglés. & \\
\hline $\begin{array}{l}\text { I5: Artículos que incluyan diseño de arquitecturas, } \\
\text { marcos de trabajo, modelos de aplicación usando } \\
\text { social media }\end{array}$ & \\
\hline
\end{tabular}

Tabla 1 - Criterios de Inclusión y Exclusión (I/E).

\subsection{Evaluación de calidad}

La evaluación de la calidad de los estudios se realizó por tres integrantes del grupo de investigación para determinar el nivel de detalle con el que cuentan los artículos considerando que: PC1 ¿el artículo explica claramente una o varias técnicas y/o herramientas de social media? y PC2 ¿̇el artículo explica el desarrollo de una arquitectura de software con social media?

La puntuación para los criterios se asignó de acuerdo con la escala de o - 1; donde o $=$ No cumple en absoluto, $0.5=$ Cumple parcialmente y $1=$ Cumple totalmente. Los valores de calidad obtenidos variaron entre 0.5 y 0.75 y un promedio de 0.50 (Tabla 2). Como último filtro, se seleccionaron los estudios con un indicador de calidad mayor o igual a 0.05 .

\begin{tabular}{lccc}
\hline Autor(es) & PC1 & PC2 & $\overline{\mathbf{X}}$ \\
\hline Younis, E. (2015) & 1 & 0 & 0.5 \\
\hline Shen \& Kuo (2015) & 1 & 0 & 0.5 \\
\hline Immonen et al. (2015) & 0.5 & 1 & 0.75 \\
\hline Gupta \& Gupta (2016) & 0.5 & 0 & 0.25 \\
\hline Raspopović et al. (2016) & 0.5 & 0 & 0.75 \\
\hline Buxarrais, M. R. (2016) & 0.5 & 0 & 0.25 \\
\hline Adinolfi et al. (2016) & 0.5 & 1 & 0.75 \\
\hline
\end{tabular}




\begin{tabular}{lccc}
\hline Autor(es) & PC1 & PC2 & $\overline{\mathbf{X}}$ \\
\hline Hu \& Chen (2016) & 0.5 & 0 & 0.25 \\
\hline Lipizzi et al. (2016) & 1 & 0 & 0.5 \\
\hline Peruta \& Shields (2017) & 1 & 0 & 0.5 \\
\hline Salloum et al. (2017) & 1 & 0 & 0.5 \\
\hline He et al. (2017) & 1 & 0 & 0.5 \\
\hline Chugh \& Ruhi (2018) & 1 & 0 & 0.5 \\
\hline Mushtaq et al. (2018) & 0.5 & 1 & 0.75 \\
\hline Stieglitz et al.(2018) & 1 & 0 & 0.5 \\
\hline Kurnia \& Suharjito (2018) & 1 & 0.5 & 0.75 \\
\hline Amin \& Rajadurai (2018) & 1 & 0 & 0.5 \\
\hline Hwa Choi, S. (2019) & 0.5 & 0 & 0.25 \\
\hline Peláez et al. (2019) & 0.5 & 1 & 0.75 \\
\hline Arora et al. (2019) & 0.5 & 0 & 0.25 \\
\hline Hayat et al. (2019) & 0.5 & 1 & 0.75 \\
\hline Tocoglu et al. (2019) & 0.5 & 0 & 0.25 \\
\hline Goyal \& Goyal (2020) & 1 & 0 & 0.5 \\
\hline Han et al. (2020) & 1 & 0 & 0.5 \\
\hline Noor \& Haneef (2020) & 1 & 0 & 0.5 \\
\hline Purohit, Castillo \& Pandey (2020) & 0.5 & 0.5 & 0.5 \\
\hline Arafeh et al. (2020) & 1 & 0 & 0.5 \\
\hline Al-Maatouk et al. (2020) & 1 & 0.5 \\
\hline Bitiktas \& Tuna (2020) & 1 & 0.5 \\
\hline Meng et al. (2020) & 0.5 \\
\hline & 1 & 0 \\
\hline
\end{tabular}

Tabla 2 - Evaluación de calidad de los estudios primarios

\subsection{Amenazas a la validez}

A continuación, se presentan las amenazas a la validez del estudio, así como las estrategias para minimizarlas (Kitchenham, 2007; Ramírez-Alpízar et al., 2020).

Cobertura sujeta a los términos de búsqueda y a los repositorios de búsqueda. Las bases de datos seleccionadas son reconocidas por tener gran cobertura en la disciplina de Ingeniería de software. La cadena de búsqueda utilizada en este estudio fue definida a partir de una búsqueda exploratoria en bases de datos y un conjunto de artículos base. Durante el proceso de I/E, si existían dudas sobre un artículo específico, se procedió a su lectura completa.

Extracción y clasificación. El proceso de extracción de artículos y la clasificación de los mismos fue realizado por el primer autor. La interpretación de los resultados por parte de los demás autores y la amenaza a la validez primero y segundo autor. Los artículos fueron clasificados de acuerdo con lo reportado por los autores originales y 
en caso de no ser reportados explícitamente los autores de este estudio les asignaron una clasificación.

\subsection{Proceso de extracción y análisis}

En este proceso se extrajeron los datos para su identificación y la información necesaria para responder las preguntas PI1 y PI2. Para ello, se agruparon los estudios primarios en cuatro categorías: 1) Enfoques, 2) Modelos de análisis para social media, 3) Diseño de arquitecturas de software y 4) Técnicas/Herramientas de social media, donde las categorías 1 y 2 se asociaron a PI1 y las categorías 3 y 4 a PI2. A cada artículo se le asignó una etiqueta conforme al área de aplicación: Educación, Mercadotecnia, Gestión de negocios e Investigación. Las áreas de aplicación definidas identificaron aquellos estudios que validaban sus propuestas por medio de estudios empíricos y analizaban aplicaciones de las técnicas, herramientas y modelos de arquitecturas de software. Finalmente, se realizó una conclusión a partir del análisis y resultados obtenidos en la búsqueda.

\section{Análisis de resultados}

En esta sección, se presentan los resultados del mapeo de literatura realizado. En la Figura 2 se presenta la distribución de los estudios primarios dentro del periodo de 2015 a 2020. El 10\% corresponde al año 2015, el 20\% en el año 2016, el 10\% en el año 2017, un $16.7 \%$ en el 2018 y 2019 y el 26.6\% en el año 2020. El 36.67\% de los estudios fueron obtenidos de la base de datos Elsevier, mientras que las bases de datos con menor porcentaje de estudios seleccionados fueron Scopus y Springer con 10\% cada una. El 16.67\% se obtuvieron de IEL y el $26.66 \%$ pertenece a otras fuentes como AMC, Wiley, Emerald insight y Google Scholar.

\subsection{Enfoques y modelos de análisis para social media}

Para dar respuesta a la primera PI1, se identificaron aquellos artículos que presentaron un marco conceptual centrado a social media o una implementación de un caso de estudio o escenarios de prueba. En la Tabla 3 se identifican los 8 artículos representando un $\mathbf{2 6 . 6 7 \%}$ del total de los estudios primarios, de los cuales 5 pertenecen al área de Investigación y 3 al área de educación; denotando el interés en presentar definiciones que ayuden a interpretar y adaptar los conceptos de social media. Autores como Gupta \& Gupta (2016), Stieglitz et al. (2018) y Han et al. (2020) presentan revisiones de conceptos, tales como: Analítica de social media (Social media analytics), Análisis de sentimientos (Sentiment Analysis) y Minería de opiniones (Opinion Mining).

La analítica de social media se refiere al campo de investigación interdisciplinario emergente que tiene como objetivo combinar, ampliar y adaptar métodos para el análisis de datos de redes sociales (Stieglitz et al., 2018). Por otro lado, el análisis de sentimientos es una forma particular de $\mathrm{SMM}^{2}$ que implica el uso de una variedad de tecnologías para determinar los sentimientos personales expresados con respecto a temas particulares en

\footnotetext{
${ }^{2}$ La monitorización de redes sociales (SMM por sus siglas en inglés) es el proceso de extraer conocimiento útil o procesable de estos datos generados por usuarios a gran escala del ámbito de las redes sociales.
} 
las plataformas de redes sociales con el fin de medir el ambiente o el sentimiento general (Gupta \& Gupta, 2016).

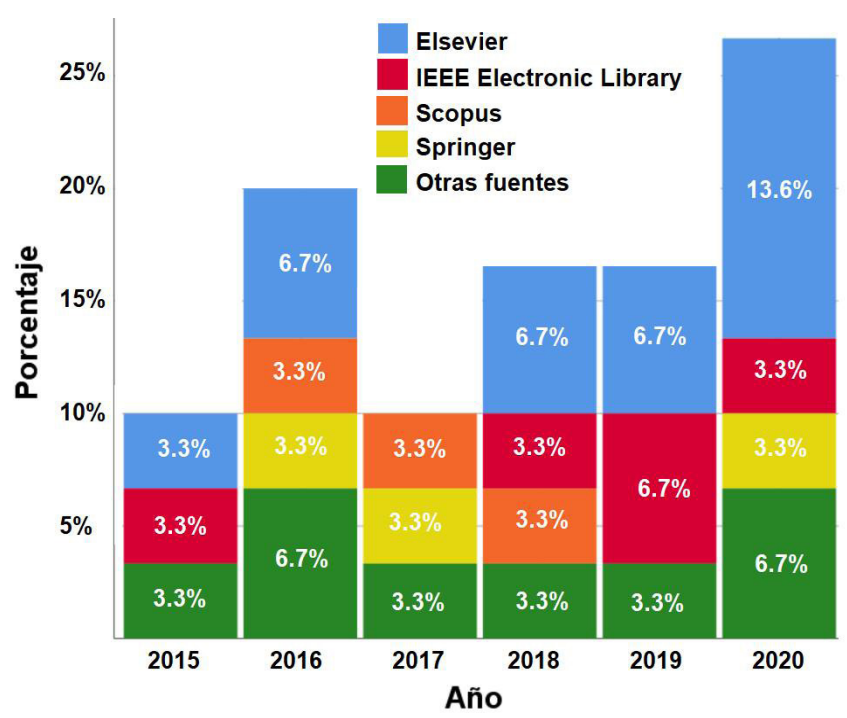

Figura 2 - Distribución de estudios primarios por año y base de datos.

\begin{tabular}{llc}
\hline $\begin{array}{l}\text { Área de } \\
\text { aplicación }\end{array}$ & Estudios con enfoques de social media & Cantidad \\
\hline Educación & Buxarrais, M. R. (2016), Chugh \& Ruhi (2018), Amin \& Rajadurai (2018) & 3 \\
\hline Investigación & $\begin{array}{l}\text { Gupta \& Gupta (2016), Stieglitz et al. (2018), Goyal \& Goyal (2020), Han } \\
\text { et al. (2020), Meng et al. (2020) }\end{array}$ & 5 \\
\hline
\end{tabular}

Tabla 3 - Número de estudios de la categoría enfoques de social media.

El utilizar los argumentos basados en el comportamiento de los likes/reacciones de los usuarios, representa la fuerza del vínculo social en un contexto de comportamiento de rebaño y proporciona una visión sobre el comportamiento de los usuarios de las redes sociales y la influencia social (Meng et al., 2020). Así mismo, la Minería de opiniones se ocupa de analizar las opiniones, criticas, actitudes y emociones de las personas hacia diferentes marcas, empresas, productos e incluso individuos usando algoritmos de preprocesamiento de texto (Rousidis et al., 2020).

En el desarrollo y propuestas de modelos de análisis es imperativo que los administradores de redes sociales implementen estrategias que conduzcan a la participación en las publicaciones para maximizar la visibilidad y el compromiso de los usuarios (Peruta \& Shields, 2017). Con respecto a conocer los modelos de análisis de social media, se recopilaron artículos que presentaron la descripción de uno o varios de los procesos de social media: descubrimiento de los datos (Mushtaq et al., 2018), 
recopilación, preparación o análisis de datos (Goyal \& Goyal, 2020; Gupta \& Gupta, 2016). Autores como Goyal \& Goyal (2020), Meng et al. (2020) y Liu et al. (2016), confirman el proceso de análisis de datos con cuatro actividades básicas, y sitúan los datos generados por las redes sociales como parte del Big data. Así mismo, resaltan la importancia durante el análisis de estos, debido a que la mayor parte de los desafíos por parte de los investigadores aún se encuentran durante esta etapa (Liu et al., 2016). Dichas propuestas de modelos de análisis incluyen modelos de flujo de datos y/o de actividades; modelos de rol y/o acción; o modelos de flujo de trabajo. Por lo anterior, se observa que aún existen discrepancias entre los conceptos y enfoques que proponen cada uno de los autores.

Los modelos propuestos por autores como He et al. (2017); Salloum et al. (2017) y Tocoglu et al. (2019) se basan en la extracción de conocimiento de los conjuntos de datos, para posteriormente analizarlos con el objetivo de comprender no solo comportamientos, sino también sentimientos y emociones. Así mismo, existen otros autores que el enfoque de sus investigaciones se proyectan hacia la Mercadotecnia o Gestión de negocios, proponiendo soluciones para la indexación de influencers, o nichos de oportunidad para incursionar dentro de Internet (Arora et al., 2019), mencionan estrategias de una publicación eficaz dentro de las plataformas de redes sociales e identifican el tipo de mensajes o los tipos de transporte de mensaje apropiados (Bitiktas \& Tuna, 2020). Kurnia \& Suharjito (2018) y Purohit, Castillo \& Pandey (2020) desarrollaron aplicaciones basadas en sus propuestas de modelo con el propósito de realizar un monitoreo del desempeño de las publicaciones en las redes sociales, reduciendo el tiempo de espera para el conocimiento de las métricas totales de páginas de Facebook o Twitter.

La Tabla 4 identifica los 11 artículos seleccionados en la categoría de modelos de análisis de social media clasificados por área de aplicación. Cabe destacar que el 64\% de los estudios corresponde con modelos de análisis social, derivados de estudios empíricos, y no propiamente de modelos teóricos de investigación.

\begin{tabular}{|c|c|c|}
\hline $\begin{array}{l}\text { Área de } \\
\text { aplicación }\end{array}$ & Estudios con modelos de análisis de social media & Cantidad \\
\hline Educación & $\begin{array}{l}\text { Peruta \& Shields (2017) - Strategies and engagement by school type } \\
\text { Salloum et al. (2017) - Natural Language Processing (NLP) Model } \\
\text { He et al. (2017) - Topic models, Linear discriminant analysis (LDA) y } \\
\text { Probabilistic latent semantic analysis (PLSA). } \\
\text { Tocoglu et al. (2019) - Modelo de emociones básicas de Paul Ekman, } \\
\text { Artificial Neural network (ANN), Convolutional Neural Network (CNN) y } \\
\text { Recurrent Neural Network (RNN) }\end{array}$ & 4 \\
\hline Mercadotecnia & $\begin{array}{l}\text { Hu \& Chen (2016) - Helpfulness model based on prediction models } \\
\text { Arora et al. (2019) - KNN Regression y Support Vector Regression } \\
\text { Bitiktas \& Tuna (2020) - Word-of-Mouth (WOM) psychological } \\
\text { motivation }\end{array}$ & 3 \\
\hline $\begin{array}{l}\text { Gestión de } \\
\text { negocios }\end{array}$ & $\begin{array}{l}\text { Kurnia \& Suharjito (2018) - Naive Bayes, SVM y Decision Tree } \\
\text { Purohit, Castillo \& Pandey (2020) - Serviceability model (Social-EOC) }\end{array}$ & 2 \\
\hline Investigación & $\begin{array}{l}\text { Lipizzi et al. (2016) - Semantic clusters y Relation networks } \\
\text { Hwa Choi, S. (2019) - Sentiment modeling and the quantification of } \\
\text { sentiment indices used in Social Big Board }\end{array}$ & 2 \\
\hline
\end{tabular}

Tabla 4 - Identificación de Modelos de análisis de social media. 


\subsection{Técnicas y/o herramientas de social media utilizadas en el diseño de Arquitecturas de software}

La categoría de Diseño de arquitecturas de Software responde cuáles son las técnicas y/o herramientas de social media. Se identificaron 6 estudios primarios (20\%) donde la mayoría de ellos están enfocados al área de Investigación (Tabla 5).

\begin{tabular}{llc}
\hline $\begin{array}{l}\text { Área de } \\
\text { aplicación }\end{array}$ & Estudios con diseños de arquitecturas & Cantidad \\
\hline Educación & $\begin{array}{l}\text { Raspopović et al. (2016) - Arquitectura orientada a servicios } \\
\text { Adinolfi et al. (2016) - Arquitectura orientada a componentes }\end{array}$ & 2 \\
\hline $\begin{array}{l}\text { Gestión de } \\
\text { negocios }\end{array}$ & Immonen et al. (2015) - Arquitectura orientada a componentes & 3 \\
\hline Investigación & $\begin{array}{l}\text { Mushtaq et al. (2018) - Arquitectura en capas } \\
\text { Hayat et al. (2019) - Arquitecturas de aprendizaje profundo }\end{array}$ & 3 \\
\hline
\end{tabular}

Tabla 5 - Número de estudios de la categoría Diseño de arquitecturas de software.

En el diseño de arquitecturas de software se consideró la necesidad de recopilar información de la participación de los usuarios, la transparencia en los datos y el riesgo en la predicción de sentimientos y el análisis de emociones y opiniones (Mushtaq et al., 2018). Raspopović et al. (2016), presenta el diseño y los requisitos tecnológicos de una arquitectura orientada a servicios para la integración de un sistema institucional de e-learning, un sistema de gestión educativa y un entorno de aprendizaje social, centrados en promover la enseñanza y el aprendizaje. Sin embargo, cuenta con un limitado número de usuarios con acceso al conjunto de herramientas y funcionalidades. Por otro lado, Hayat et al. (2019) presenta una arquitectura de Aprendizaje profundo para evaluar y administrar la calidad de los datos en cada fase de procesamiento. La solución propuesta opera en función a los datos en tiempo real de los usuarios, donde la información del cliente se extrae de los datos de las redes sociales para determinar la satisfacción con respecto a la calidad de un producto. Adinolfi et al. (2016), Immonen et al. (2015) y Peláez, Martínez \& Vargas (2019) hacen énfasis en la necesidad de diseñar experimentos que permitan comprender mejor cómo determinados estados cognitivos o emocionales provocan o bloquean algún camino de aprendizaje o decisiones de los usuarios.

La categoría de Técnicas y/o herramientas de social media se enfoca en encontrar los procedimientos o recursos empleados para la aplicación de procesos de social media. Así mismo, agrupar las diversas herramientas existentes que apoyan en la implementación de social media en las redes sociales. En la Tabla 6 se especifican las técnicas y herramientas de social media para cada área de aplicación. 


\begin{tabular}{llc}
\hline $\begin{array}{l}\text { Área de } \\
\text { aplicación }\end{array}$ & Técnicas y herramientas de social media & Cantidad \\
\hline Educación & $\begin{array}{l}\text { Shen \& Kuo (2015) - Técnicas de minería de análisis de tendencias y } \\
\text { sentimientos y massive open online courses (MOOCs) } \\
\text { Al-Maatouk et al. (2020) - Task/Technology Fit (TTF) y Technology } \\
\text { acceptance model (TAM) }\end{array}$ & 2 \\
\hline Mercadotecnia & Younis, E. (2015) - Package ‘twitter’ & 1 \\
\hline $\begin{array}{l}\text { Gestión de } \\
\text { negocios }\end{array}$ & Arafeh et al. (2020) - Neo4j Graph Platform & 1 \\
\hline Investigación & Noor \& Haneef (2020) - Hama and Spark tools y Jaql & 1 \\
\hline
\end{tabular}

Tabla 6 - Identificación de técnicas y herramientas de social media.

En esta área se reconoce el uso de massive open online courses (MOOCs) para extraer la información para los análisis de datos, debido a que la disponibilidad y la popularidad de los servicios de redes sociales, valioso en opiniones, están aumentando las comunidades MOOC y muestran un análisis de sentimientos de los datos de Twitter o Facebook para proporcionar información sustancial sobre las percepciones de los usuarios (Shen \& Kuo, 2015). En la literatura, no se identificó un método estándar para extraer y analizar datos de redes sociales. Autores como Noor \& Haneef, (2020) y Younis, (2015) se enfocan en desarrollar herramientas de código abierto para minería tanto de texto, como de sentimientos, utilizando el conjunto de paquetes de lenguaje de programación $\mathrm{R}$, el cual es aplicable en diversas redes sociales. Los resultados de los autores coinciden en que los casos de prueba y los resultados experimentales enfatizan la importancia de la definición de la estrategia inicial y la aplicación apropiada de los métodos, para obtener una correcta extracción de conocimientos (Arafeh et al., 2020). La mayoría de los métodos, adaptados desde Minería de datos, Big data o machine learning, apuntan a que social media apoyen las actividades de comercialización de productos o marcas, especialmente durante las primeras etapas de su incorporación en el mercado (Han et al., 2020).

Los procesos descritos son expuestos desde el criterio de los datos en bruto en las redes sociales, para después realizar alguna clasificación determinada por el interés particular del autor. En los diseños de las arquitecturas se ha implementado un proceso de localización de fuentes de información mediante Web crawlers, siempre se hace uso de una red social como servicio de verificación a través de su API, la cual se encuentra integrada en el sistema y posteriormente se analizan los datos utilizando la librería Python NLTK. Expresan que el apoyarse en métodos de Aprendizaje profundo para desarrollar arquitecturas de software ayuda al representar datos en plataformas social media con multidominio, enfocadas en el análisis de comportamiento, análisis de negocios, análisis de sentimientos y la detección de anomalías. Coincidiendo en que la 
solución arquitectónica debe permitir insertar nuevas fuentes y conjuntos de datos para la extracción de información, así como nuevas métricas y algoritmos para la evaluación de los datos.

\subsection{Análisis del conjunto de artículos}

Las implicaciones prácticas y de investigación se relacionan en el aporte de definir un marco de referencia para el desarrollo de software, con arquitecturas de software adecuadas para aprovechar los datos en los social media. La Figura 3 presenta el gráfico de dispersión de puntos que expresa las categorías y áreas de aplicación ordenadas por años. Existe una concentración del 37\% dentro de la categoría 2 (Modelos de análisis social media), los cuales el 36.37\% se enfocan al área 1 (Educación). Se observó una mayor incidencia en el área 4 (Investigación) con un 40\%, independientemente de cual fuera la categoría asignada al estudio. Existen estudios que comparten características tanto en categoría, como en área de aplicación. Esto demostró que, pese a que existen diversos centros de investigación de procedencia de los estudios, existe una congruencia en el interés en un mismo tópico por parte de los diferentes autores. El acumulado en los estudios pertenecientes a los últimos tres años fue del 57\%. Se identificó que el $43.33 \%$ de los artículos corresponden a los años 2019 y 2020, validando el interés en los enfoques de social media como un tema o línea de investigación actual.

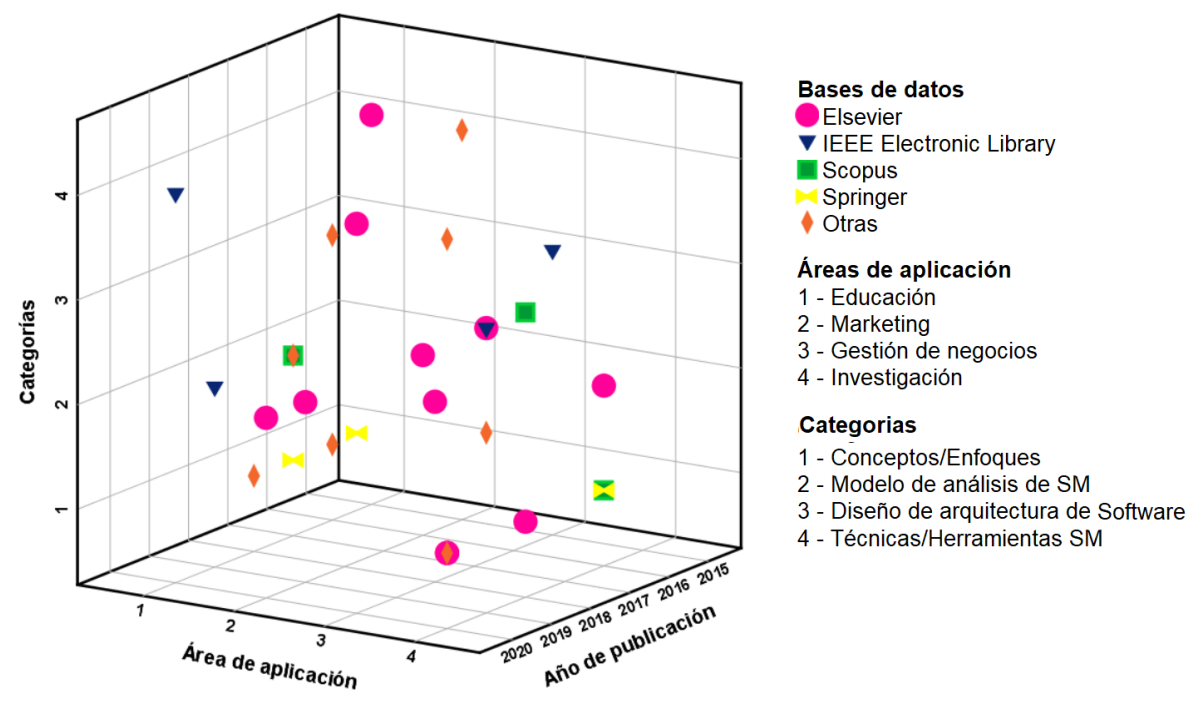

Figura 3 - Gráfico de dispersión de puntos correspondiente a los estudios primarios.

\section{Conclusiones}

Este estudio reporta los resultados de un mapeo sistemático de literatura sobre las técnicas y herramientas de social media con la integración de arquitecturas de software. Se analizó un conjunto de 30 estudios primarios publicados entre 2015 y 
2020 encontrando un panorama teórico y resultados reportados de las prácticas y áreas de aplicación comunes y las categorías planteadas. Se observó el beneficio de adoptar estas técnicas y herramientas, y se detectó que las organizaciones han mostrado un crecimiento en el interés de incorporarlas en sus procesos cotidianos. Sin embargo, aún existen retos en la adopción de éstas. En relación a las limitaciones del estudio, cabe destacar que los resultados se han valorado de forma conjunta, no realizando un análisis multinivel con respecto a las herramientas y técnicas presentadas.

Los trabajos analizados aún están con un criterio orientado al proceso de Knowledge Discovery in Databases, propio de Minería de datos, por lo que se identifica la oportunidad para proponer marcos de trabajo, marcos de referencia o metodologías enfocados en social media. En este sentido faltan esquemas de trabajo que apoyen en la orientación y trazabilidad de actividades, y artefactos pertenecientes a un proceso de social media, debido a que no abordan la temática desde el punto de vista de elementos de procesos de software.

Se identificó que las áreas de Mercadotecnia y Gestión de negocios son las de mayor uso, lo que brinda la posibilidad de crear modelos orientados al ámbito de educación para el análisis del comportamiento de los estudiantes al utilizar las redes sociales con fines de aprendizaje. Esto enriquecería la actividad académica, pero sobre todo el brindar información asertiva para la toma de decisiones o el planteamiento de estrategias. Actualmente, se está realizando una investigación sobre la selección y aplicación de algunas técnicas y herramientas de social media para el diseño de una arquitectura de software de una plataforma digital educativa.

\section{Agradecimientos}

Se agradece al Consejo Nacional de Ciencia y Tecnología (CONACYT) por el apoyo económico brindado al primer autor.

\section{Referencias}

Adinolfi, P., D’Avanzo, E., Lytras, M. D., Novo-Corti, I., \& Picatoste, J. (2016). Sentiment Analysis to Evaluate Teaching Performance. International Journal of Knowledge Society Research, 7(4), 86-107. https://doi.org/10.4018/ijksr.2016100108

Al-Maatouk, Q., Othman, M. S., Aldraiweesh, A., Alturki, U., Al-Rahmi, W. M., \& Aljeraiwi, A. A. (2020). Task-technology fit and technology acceptance model application to structure and evaluate the adoption of social media in academia. IEEE Access, 8, 78427-78440. https://doi.org/10.1109/ACCESS.2020.2990420

Amin, A., \& Rajadurai, J. (2018). The Conflict between Social Media and Higher Education Institutions. Global Business and Management Research: An International Journal, 1O(4).

Arafeh, M., Ceravolo, P., Mourad, A., Damiani, E., \& Bellini, E. (2020). Ontology based recommender system using social network data. Future Generation Computer Systems, 115, 769-779. https://doi.org/10.1016/j.future.2020.09.030 
Arora, A., Bansal, S., Kandpal, C., Aswani, R., \& Dwivedi, Y. (2019). Measuring social media influencer index- insights from facebook, Twitter and Instagram. Journal of Retailing and Consumer Services, 49(2), 86-101. https://doi.org/10.1016/j. jretconser.2019.03.012

Batrinca, B., \& Treleaven, P. C. (2015). Social media analytics: a survey of techniques, tools and platforms. AI and Society, 3O(1), 89-116. https://doi.org/10.1007/ s00146-014-0549-4

Bitiktas, F., \& Tuna, O. (2020). Social media usage in container shipping companies: Analysis of Facebook messages. Research in Transportation Business and Management, 34(February). https://doi.org/10.1016/j.rtbm.2020.100454

Brenes Carranza, J. A., Martínez Porras, A., Quesada López, C. U., \& Jenkins Coronas, M. (2020). Sistemas de apoyo a la toma de decisiones que usan inteligencia artificial en la agricultura de precisión. RISTI - Revista Ibérica de Sistemas y Tecnologías de La Información, 28(18), 217-229. http://risti.xyz/issues/ristie28.pdf

Buxarrais, M. R. (2016). Redes sociales y educación Social networks and education. Education in the Knowledge Society, 17(2), 15-20.

Chugh, R., \& Ruhi, U. (2018). Social media in higher education: A literature review of Facebook. Education and Information Technologies, 23(2), 605-616. https://doi.org/10.1007/s10639-017-9621-2

Diamantini, C., Mircoli, A., Potena, D., \& Storti, E. (2019). Social information discovery enhanced by sentiment analysis techniques. Future Generation Computer Systems, 95, 816-828. https://doi.org/10.1016/j.future.2018.01.051

Gonzalez, C., \& Munoz, L. (2016). Online Social networks its impact on higher Education: Case Study of Technological University of Panama. Campus Virtuales, 5(1), 84-90.

Goyal, S. J., \& Goyal, R. (2020). Social Media Analytics - Challenges during the Data Analysis. Artificial \& Computational Intelligence, 1(2). https://acors.org/ijacoi/ VOL1_ISSUE2_10.pdf

Gupta, V., \& Gupta, M. (2016). Social Media Mining: A New Framework and Literature Review. International Journal of Business Analytics, 3(1), 58-68. https://doi.org/10.4018/IJBAN.2016010104

Han, J., Park, D., Forbes, H., \& Schaefer, D. (2020). A computational approach for using social networking platforms to support creative idea generation. Procedia CIRP, 91, 382-387. https://doi.org/10.1016/j.procir.2020.02.190

Hayat, M. K., Daud, A., Alshdadi, A. A., Banjar, A., Abbasi, R. A., Bao, Y., \& Dawood, H. (2019). Towards Deep Learning Prospects: Insights for Social Media Analytics. IEEE Access, 7, 36958-36979. https://doi.org/10.1109/ACCESS.2019.2905101

He, Y., Wang, C., \& Jiang, C. (2017). Mining Coherent Topics With Pre-Learned Interest Knowledge in Twitter. IEEE Access, 5, 10515-10525. https://doi.org/10.1109/ ACCESS.2017.2696558 
Herrera, H. (2012). Social Networks: A new difusion tool. Reflexiones, 91(2), 121-128. https://revistas.ucr.ac.cr/index.php/reflexiones/article/view/1513/1521

Hu, Y. H., \& Chen, K. (2016). Predicting hotel review helpfulness: The impact of review visibility, and interaction between hotel stars and review ratings. International Journal of Information Management, 36(6), 929-944. https://doi.org/10.1016/j. ijinfomgt.2016.06.003

Hwa Choi, S. (2019). Development of a Disaster Safety Sentiment Index via Social Media Mining. Journal of Public Policy and Administration, 3(1), 29. https://doi.org/10.11648/j.jppa.20190301.14

Immonen, A., Pääkkönen, P., \& Ovaska, E. (2015). Evaluating the Quality of Social Media Data in Big Data Architecture. IEEE Access, 3, 2028-2043. https://doi.org/10.1109/ACCESS.2015.2490723

Injadat, M., Salo, F., Nassif, A. B., Injadat, M., Salo, F., \& Nassif, A. B. (2016). Data Mining Techniques in Social Media: A Survey. Neurocomputing. http://dx.doi.org/10.1016/j.neucom.2016.06.045

Kitchenham, B. (2007). Guidelines for performing Systematic Literature Reviews in Software Engineering. https://doi.org/10.1145/1134285.1134500

Kurnia, P. F., \& Suharjito. (2018). Business Intelligence Model to Analyze Social Media Information. Procedia Computer Science, 135, 5-14. https://doi.org/10.1016/j. procs.2018.08.144

Lara, T. (2008). La nueva esfera pública: los medios de comunicación como redes sociales. Telos: Cuadernos de Comunicación e Innovación, 76, 128-131.

Lipizzi, C., Dessavre, D. G., Iandoli, L., \& Marquez, J. E. R. (2016). Social media conversation monitoring: Visualize information contents of twitter messages using conversational metrics. Procedia Computer Science, 8o, 2216-2220. https://doi.org/10.1016/j.procs.2016.05.384

Liu, H., Morstatter, F., Tang, J., \& Zafarani, R. (2016). The good, the bad, and the ugly: uncovering novel research opportunities in social media mining. International Journal of Data Science and Analytics, 1(3-4), 137-143. https://doi.org/10.1007/ S41060-016-0023-0

Maier, M. W., Emery, D., \& Hilliard, R. (2001). Software architecture: Introducing IEEE standard 1471. Computer, 34(4), 107-109. https://doi.org/10.1109/2.917550

Martín Blanco, V. (2015). Social Media: Herramientas de gestión para el community manager. In Fundación Confemetal (1ra ed., Vol. 1).

Meng, X., Shi, L., Yao, L., Zhang, Y., \& Cui, L. (2020). Herd Behavior in Social Media: The Role of Facebook Likes, Strength of Ties, and Expertise. Information \& Management, Iii, 124658. https://doi.org/10.1016/j.colsurfa.2020.124658 
Mushtaq, H., Malik, B. H., Shah, S. A., Siddique, U. B., Shahzad, M., \& Siddique, I. (2018). Implicit and explicit knowledge mining of Crowdsourced communities: Architectural and technology verdicts. International Journal of Advanced Computer Science and Applications, 9(1), 105-111. https://doi.org/10.14569/IJACSA.2018.090114

Noor, M. N., \& Haneef, F. (2020). A Review on Big Data and Social Network Analytics Techniques. Researchpedia Journal of Computing, 1(1), 39-49.

Peláez, J. I., Martínez, E. A., \& Vargas, L. G. (2019). Decision making in social media with consistent data. Knowledge-Based Systems, 172, 33-41. https://doi.org/10.1016/j. knosys.2019.02.009

Peruta, A., \& Shields, A. B. (2017). Social media in higher education: understanding how colleges and universities use Facebook. Journal of Marketing for Higher Education, 27(1), 131-143. https://doi.org/10.1080/08841241.2016.1212451

Petersen, K., Vakkalanka, S., \& Kuzniarz, L. (2015). Guidelines for conducting systematic mapping studies in software engineering: An update. Information and Software Technology, 64, 1-18. https://doi.org/10.1016/j.infsof.2015.03.007

Purohit, H., Castillo, C., \& Pandey, R. (2020). Ranking and grouping social media requests for emergency services using serviceability model. Social Network Analysis and Mining, 1O(1). https://doi.org/10.1007/s13278-020-0633-3

Ramírez-Alpízar, A., Jenkins, M., Martínez, A., \& Quesada-Lopéz, C. (2020). Uso de técnicas de minería de datos y aprendizaje automático para la detección de fraudes en estados financieros: un mapeo sistemático de literatura. RISTI - Revista Ibérica de Sistemas y Tecnologías de La Información, (28), 97-109.

Raspopović, M., Cvetanović, S., Stanojević, D., \& Opačić, M. (2016). Software architecture for integration of institutional and social learning environments. Science of Computer Programming, 129, 92-102. https://doi.org/10.1016/j. scico.2016.07.001

Rousidis, D., Koukaras, P., \& Tjortjis, C. (2020). Social media prediction: a literature review. Multimedia Tools and Applications, 79(9-10), 6279-6311. https://doi.org/10.1007/s11042-019-08291-9

Sabate,F., Berbegal-Mirabent, J.,Cañabate,A., \&Lebherz,P.R.(2014).Factorsinfluencing popularity of branded content in Facebook fan pages. European Management Journal, 32(6), 1001-1011. https://doi.org/10.1016/j.emj.2014.05.001

Salloum, S. A., Al-Emran, M., Monem, A. A., \& Shaalan, K. (2017). A survey of text mining in social media: Facebook and Twitter perspectives. Advances in Science, Technology and Engineering Systems, 2(1), 127-133. https://doi.org/10.25046/ aj020115

Shen, C. W., \& Kuo, C. J. (2015). Learning in massive open online courses: Evidence from social media mining. Computers in Human Behavior, 51, 568-577. https://doi.org/10.1016/j.chb.2015.02.066 
Stieglitz, S., Mirbabaie, M., Ross, B., \& Neuberger, C. (2018). Social media analytics Challenges in topic discovery, data collection, and data preparation. International Journal of Information Management, 39, 156-168. https://doi.org/10.1016/j. ijinfomgt.2017.12.002

Tocoglu, M. A., Ozturkmenoglu, O., \& Alpkocak, A. (2019). Emotion Analysis from Turkish Tweets Using Deep Neural Networks. IEEE Access, 7, 183061-183069. https://doi.org/10.1109/ACCESS.2019.2960113

Ureña, R., Chiclana, F., Melançon, G., \& Herrera-Viedma, E. (2019). A social network based approach for consensus achievement in multiperson decision making. Information Fusion, 47, 72-87. https://doi.org/10.1016/j.inffus.2018.07.006

Verhoef, P. C., \& Lemon, K. N. (2013). Successful customer value management: Key lessons and emerging trends. European Management Journal, 31(1), 1-15. https://doi.org/10.1016/j.emj.2012.08.001

Wohlin, C., Runeson, P., Höst, M., Ohlsson, M. C., Regnell, B., \& Wesslén, A. (2012). Planning. In: Experimentation in Software Engineering. Springer. https://doi.org/10.1007/978-3-642-29044-2_8

Younis, E. (2015). Sentiment Analysis and Text Mining for Social Media Microblogs using Open Source Tools: An Empirical Study. International Journal of Computer Applications, 112(5), 975-8887. 